\title{
Update of research on the role of EZH2 in cancer progression
}

This article was published in the following Dove Press journal:

OncoTargets and Therapy

3 April 2013

Number of times this article has been viewed

\author{
Liang Shen' \\ Jing Cui ${ }^{2}$ \\ Shumei Liang ${ }^{3}$ \\ Yingxin Pang' \\ Peishu Liu' \\ 'Department of Obstetrics and \\ Gynecology, Qilu Hospital, Shandong \\ University, ${ }^{2}$ Department of Oral \\ and Maxillofacial Surgery, Jinan \\ Stomatologic Hospital, ${ }^{3}$ Department \\ of Obstetrics and Gynecology, \\ Provincial Hospital Affiliated to \\ Shandong University, Jinan, People's \\ Republic of China
}

\begin{abstract}
Accumulating evidence shows that enhancer of zeste homolog $2(\mathrm{E} 2 \mathrm{H} 2)$ is upregulated in a broad range of cancer types, such as breast cancer, prostate cancer, ovarian cancer, and colon cancer. Therefore, inhibiting EZH2 expression may be a promising strategy for anticancer therapy. This review focuses on the current understanding of the mechanisms underlying EZH2 regulation that are involved in cancer progression. Also, it introduces two EZH2 inhibitors that target EZH2 and could be potentially applied in the treatment of cancer in the future.
\end{abstract}

Keywords: EZH2, PRC2, cancer

\section{Introduction}

Enhancer of zeste homolog 2 (EZH2) encodes a histone methyltransferase, which is the catalytic core protein of the polycomb repressor complex 2 (PRC2). ${ }^{1,2}$ PRC2 is well known for initiating target gene silencing by promoting H3K27 trimethylation, which is catalyzed by EZH2. Several articles implicated that the EZH2 is involved in the cell proliferation, invasion, apoptosis, angiogenesis, and metastasis of cancer progression.

\section{Underlying mechanism of EZH2 in cancer progression}

Polycomb group proteins maintain the gene expression pattern of different cells that is set during early development by modifying chromatin structure. ${ }^{3}$ In mammals, there are two main polycomb group complexes, PRC1 and PRC2. The PRC2 complex mainly consists of four core components: EZH2, suppressor of zeste 12 homolog (SUZ12), embryonic ectoderm development protein (EED), and retinoblastoma-associated protein 46/48. EZH2 via the SET domain catalyzes H3K27me3, and is associated with the silencing of tumor suppressor genes such as DAB2IP (Figure 1). ${ }^{4}$

Cancer stem cells are a subgroup of cancer cells with several features: unlimited self-renewal potential, tumorigenicity, and chemoresistance. ${ }^{5}$ Several papers showed that signal transducer and activator of transcription 3 (STAT3) and EZH2 are involved in the self-renewal, pluripotency, and proliferation of cancer stem cells. ${ }^{6}$ Akt-dependent Ser21 phosphorylation of EZH2 has been reported in breast cancer cells treated with insulin-like growth factor 1 or estrogen. ${ }^{7}$ Furthermore, Chen et al confirmed the c-Jun N-terminal kinase (JNK) regulation of STAT3 and link the JNK-STAT3-Akt signaling axis to the phosphorylation of EZH2 ${ }^{8}$ Suva et al provided evidence that direct downregulated transcriptional regulation of c-Myc by EZH2 may constitute a novel mechanism underlying glioblastoma cancer stem cell maintenance. ${ }^{9}$
Correspondence: Peishu Liu Department of Obstetrics and Gynecology, Qilu Hospital, Shandong University, Wen Hua Xi Lu No 107. Jinan, Shandong 2500 I2, People's Republic of China

Tel +8653182169563

Fax +8653186927544

Email peishuliu1982@I63.com 


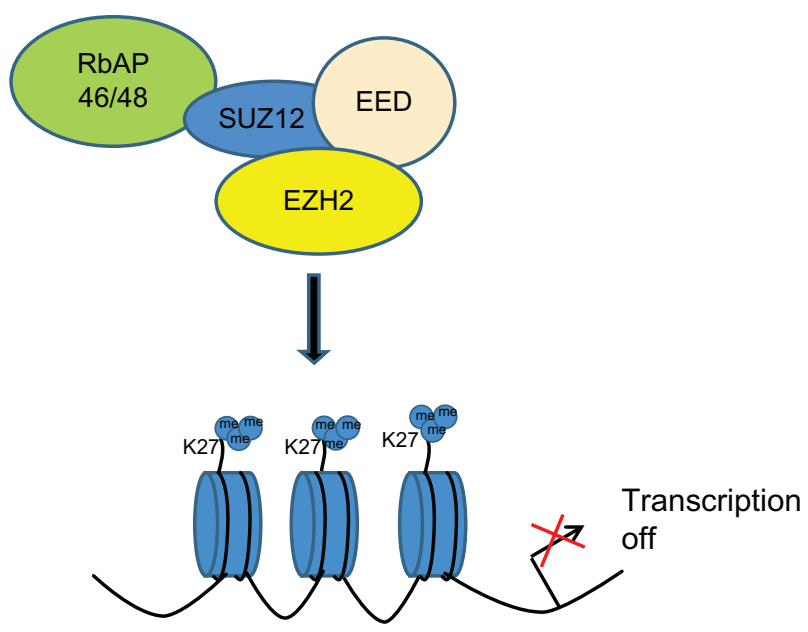

Figure I Schematic representation of transcriptional gene repression by enhancer of zeste homolog 2 (EZH2). Proposed mechanism leads to aberrantly high levels of trimethylation on histone $\mathrm{H} 3 \mathrm{~K} 27$ in cancer.

Abbreviations: EED, embryonic ectoderm development; EZH2, enhancer of zeste homolog 2; RbAP46/48, retinoblastoma-associated protein 46/48; SUZI2, suppressor of zeste 12 homolog.

\section{Roles of EZH2 in cancer}

The accumulated finding is that EZH2 levels are abnormally elevated in cancer tissues compared with corresponding normal tissues. Furthermore, higher EZH2 levels are correlated with advanced stages of disease and poor prognosis.

\section{$\mathrm{EZH} 2$ in prostate cancer}

Analyses of patient samples significantly correlate abnormally elevated EZH2 levels with increased proliferation rates, invasiveness, and metastasis of prostate cancer. Van Leenders et al showed that expression of EZH2 was significantly enhanced in tumors with a Gleason score of more than eight, extraprostatic extension, positive surgical margins, and prostate-specific antigen recurrence. ${ }^{10}$ Bryant et al found that knockdown of endogenous EZH2 reduced proliferation and invasion in prostate cancer cells. ${ }^{11}$ Furthermore, Ren et al indicated that EZH2 promoted prostate cancer cell invasion and metastasis via the repression of RKIP, a metastasis suppressor gene. ${ }^{12}$ It is known that metastasis is associated with the balance between matrix metalloproteinases and their inhibitors, ie, tissue inhibitor of metalloproteinases. Shin et al discovered that EZH2 plays an active role in this process by repressing the expression of metallopeptidase inhibitor 2 and metallopeptidase inhibitor 3 in prostate cancer cells. ${ }^{13}$ Although, there are eleven genetic variations in EZH2 in prostate cancer, genetic variations of the EZH2 gene are not responsible for the linkage of $7 q$ to aggressive prostate cancer. ${ }^{14}$ However, there is another mechanism for EZH2 in prostate cancer. $\mathrm{Xu}$ et al discovered that in the castration-resistant prostate cancer cells, EZH2 could be a transcriptional coactivator of androgen receptor instead of a transcriptional repressor of PCR $2 .{ }^{15}$ Furthermore, their study demonstrates that the phosphatidylinositol 3-kinase-Akt pathway could mediate phosphorylation of EZH2 at Ser21 and this phosphorylation could be involved in the transcriptional coactivator.

\section{$\mathrm{EZH} 2$ in breast cancer}

Overexpressed EZH2 has been reported as a biomarker of aggressive breast cancer and associated with invasion and cancer progression. ${ }^{2,16}$ EZH2 expression in 280 breast cancer patients was tested by high-density tissue microarray. EZH2 levels were elevated in patients with invasive breast carcinoma compared with normal or atypical hyperplasia. ${ }^{16}$ Furthermore, it has been shown that high levels of EZH2 are associated with poor outcome to tamoxifen therapy in advanced breast cancer. ${ }^{17,18}$ Recently, EZH2-mediated epigenetic repression of DNA damage repair in breast tumor initiating cells was identified as a mechanism that could promote expansion of breast tumor initiating cells, and may contribute to cancer progression. ${ }^{18}$ To analyze the role of EZH2 in the molecular subtypes of breast tumors (basal-like, luminal A, luminal B, human epidermal growth factor 2 (HER2)enriched, and normal-like), Holm et al tested the EHZ2 and $\mathrm{H} 3 \mathrm{~K} 27 \mathrm{me} 3$ expression in more than 400 tumors using immunohistochemistry. They found significantly high abundance of EZH2 in basal-like, triple negative, and HER2-enriched tumors, and high H3K27me3 in luminal A, HER2-enriched, and normal-like tumors. ${ }^{19}$ EZH2 overexpression inhibits breast cancer type 1 susceptibility protein (BRCA1) phosphorylation (Ser1423) and thereby promotes an increase of $\mathrm{Cdc} 25 \mathrm{C}$, an essential player for G2/M checkpoint control. ${ }^{20}$ Puppe et al observed increased EZH2 protein levels in human BRCA1-deficient tumor sections compared with other breast tumors. ${ }^{21}$ Furthermore, they used the EZH2 inhibitor 3-deazaneplanocin (DZNep) to treat the BRCA1-deficient cancer cell and BRCA1-positive cancer cells. They discovered that DZNep showed remarkable selectivity in inhibiting BRCA1-deficient tumor cells compared with BRCA1-proficient tumor cells. Their research provided another promising approach for the treatment of BRCA1-mutated breast cancers.

Besides the transcript repressor, EZH2 activation could switch to an activator via two different ways. In luminal-like estrogen receptor-positive cells, EZH2 overexpression can lead to an interaction with the Wnt signaling pathway, leading to the activation of c-Myc and cyclin D1. ${ }^{22}$ In basal-like, 
estrogen receptor-negative cells, EZH2 activates nuclear factor- $\kappa \mathrm{B}$ target genes by formation of a ternary complex with the nuclear factor- $\kappa \mathrm{B}$ components RelA and RelB. ${ }^{23}$

\section{$\mathrm{EZH} 2$ in ovarian cancer}

Several authors have indicated that EZH2 is involved with invasion and metastasis in ovarian carcinoma. Rao et al found that high expression of EZH2 was found in none of the normal ovaries, in $3 \%$ of the cystadenomas, in $23 \%$ of the borderline tumors, and in $50 \%$ of the ovarian carcinomas. ${ }^{24}$ Drug resistance is a major clinical obstacle for ovarian cancer therapy. Rizzo et al found that EZH2 played a key role in the maintenance of a drug-resistant, tumor-sustaining subpopulation of cells in ovarian cancers undergoing chemotherapy. ${ }^{25}$ Furthermore, ALDH1A1 - a putative marker for epithelial ovarian cancer stem cells - is found as a novel EZH2 target gene in epithelial ovarian cancer cells. ${ }^{26}$ Lu et al identified EZH2 as a key regulator of tumor angiogenesis in ovarian cancer. ${ }^{27}$ In endothelial cells, vascular endothelial growth factor stimulation could lead to increased expression of E2F transcription factors, which directly mediate EZH2 levels. Then, EZH2 causes the silence of VASH1 - an antiangiogenic gene - and subsequently increases angiogenesis.

\section{$\mathrm{EZH} 2$ in non-small-cell lung cancers}

Kikuchi et al analyzed the immunohistochemical assessment of 157 surgically resected non-small-cell lung cancers. ${ }^{28}$ They found that high EZH2 expression significantly correlated with non-adenocarcinoma histology, moderate and poor differentiation, advanced pathologic tumor classification, and high Ki-67 and cyclin E. Furthermore, Huqun et al found that positive EZH2 expression was associated significantly with larger tumor size in non-small-cell lung cancer. ${ }^{29}$ KaplanMeier survival analyses and logrank tests demonstrated that patients whose samples were classified into the positive EZH2 expression group had a significantly shorter overall survival. The mechanisms of EZH2 in the progression of non-small-cell lung cancer are not clear. A recent published paper shows that mir-138 - a novel tumor suppressor microribonucleic acid - could bind to 3 '-untranslated region of EZH2 and suppress the expression of EZH2 at both messenger ribonucleic acid and protein levels. ${ }^{30}$

\section{Potential cancer therapy function}

It is known that EZH2 plays an important role in cancer development. EZH2 expression can be reduced with S-adenosylhomocysteine hydrolase inhibitor DZNep, which inhibits methyltransferases and induces degradation of EZH2, SUZ12, and EED as well as the associated $\mathrm{H} 3 \mathrm{~K} 27 \mathrm{me} 3$. DZNep treatment was shown to result in reactivation of EZH2 repressed target genes, inhibited cell growth, and reduced tumor formation in various cancers. ${ }^{31}$ For example, Fiskus et al found that treatment with DZNep induced p16, p21, p27, and F-box protein 32 while depleting cyclin $\mathrm{E}$ and homeobox A9 levels in the cultured human acute myeloid leukemia cells and in primary acute myeloid leukemia cells. ${ }^{32}$ Furthermore, combined with gemcitabine, DZNep synergistically enhanced the antiproliferative activity of gemcitabine, reduced the percentage of cells in the G2/M phase, and significantly increased apoptosis. ${ }^{33}$ Recently, McCabe et al discovered a potent, highly selective, S-adenosylmethionine-competitive, small molecule inhibitor called GSK126. GSK126 can effectively inhibit the proliferation of EZH2 mutant diffuse large B-cell lymphoma cell lines and significantly inhibit the growth of EZH2 mutant diffuse large B-cell lymphoma xenografts in mice. ${ }^{34}$

\section{Conclusion}

This review highlights that overexpression of EZH2 is correlated with cell proliferation, invasion, adhesion, and metastasis in several cancer types. Inhibitors of EZH2, including DZNep and GSK126, seem very promising anticancer agents to help reach the ultimate goal of cancer prevention and remission.

\section{Disclosure}

The authors report no conflicts of interest in this work.

\section{References}

1. Cao R, Wang L, Wang H, et al. Role of histone H3 lysine 27 methylation in polycomb-group silencing. Science. 2002;298(5595):1039-1043.

2. Kunju LP, Cookingham C, Toy KA, Chen W, Sabel MS, Kleer CG. EZH2 and ALDH-1 mark breast epithelium at risk for breast cancer development. Mod Pathol. 2011;24(6):786-793.

3. Margueron R, Reinberg D. The polycomb complex PRC2 and its mark in life. Nature. 2011;469(7330):343-349.

4. Min J, Zaslavsky A, Fedele G, et al. An oncogene-tumor suppressor cascade drives metastatic prostate cancer by coordinately activating Ras and nuclear factor-кB. Nat Med. 2010;16(3):286-294.

5. Crea F, Mathews LA, Farrar WL, Hurt EM. Targeting prostate cancer stem cells. Anticancer Agents Med Chem. 2009;9(10):1105-1113.

6. Chang CJ, Hung MC. The role of EZH2 in tumour progression. Br J Cancer. 2012;106(2):243-247.

7. Cha TL, Zhou BP, Xia W, et al. Akt-mediated phosphorylation of EZH2 suppresses methylation of lysine 27 in histone H3. Science. 2005; 310(5746):306-310.

8. Chen B, Liu J, Chang Q, Beezhold K, Lu Y, Chen F. JNK and STAT3 signaling pathways converge on Akt-mediated phosphorylation of EZH2 in bronchial epithelial cells induced by arsenic. Cell Cycle. 2013;12(1):112-121.

9. Suva ML, Riggi N, Janiszewska M, et al. EZH2 is essential for glioblastoma cancer stem cell maintenance. Cancer Res. 2009;69(24) 9211-9218 
10. van Leenders GJ, Dukers D, Hessels D, et al. Polycomb-group oncogenes EZH2, BMI1, and RING1 are overexpressed in prostate cancer with adverse pathologic and clinical features. Eur Urol. 2007; 52(2):455-463.

11. Bryant RJ, Cross NA, Eaton CL, Hamdy FC, Cunliffe VT. EZH2 promotes proliferation and invasiveness of prostate cancer cells. Prostate. 2007;67(5):547-556.

12. Ren G, Baritaki S, Marathe H, et al. Polycomb protein EZH2 regulates tumor invasion via the transcriptional repression of the metastasis suppressor RKIP in breast and prostate cancer. Cancer Res. 2012;72(12):3091-3104.

13. Shin YJ, Kim JH. The role of EZH2 in the regulation of the activity of matrix metalloproteinases in prostate cancer cells. PLoS One. 2012;7(1):e30393.

14. Bachmann N, Hoegel J, Haeusler J, et al. Mutation screen and association study of EZH2 as a susceptibility gene for aggressive prostate cancer. Prostate. 2005;65(3):252-259.

15. $\mathrm{Xu} \mathrm{K}, \mathrm{Wu} \mathrm{ZJ}$, Groner $\mathrm{AC}$, et al. EZH2 oncogenic activity in castration-resistant prostate cancer cells is polycomb-independent. Science. 2012;338(6113):1465-1469.

16. Kleer CG, Cao Q, Varambally S, et al. EZH2 is a marker of aggressive breast cancer and promotes neoplastic transformation of breast epithelial cells. Proc Natl Acad Sci U S A. 2003;100(20):11606-11611.

17. Reijm EA, Jansen MP, Ruigrok-Ritstier K, et al. Decreased expression of EZH2 is associated with upregulation of ER and favorable outcome to tamoxifen in advanced breast cancer. Breast Cancer Res Treat. 2011;125(2):387-394.

18. Stefansson OA, Esteller M. EZH2-mediated epigenetic repression of DNA repair in promoting breast tumor initiating cells. Breast Cancer Res. 2011;13(3):309.

19. Holm K, Grabau D, Lovgren K, et al. Global H3K27 trimethylation and EZH2 abundance in breast tumor subtypes. Mol Oncol. 2012; 6(5):494-506.

20. Gonzalez ME, Li X, Toy K, et al. Downregulation of EZH2 decreases growth of estrogen receptor-negative invasive breast carcinoma and requires BRCA1. Oncogene. 2009;28(6):843-853.

21. Puppe J, Drost R, Liu X, et al. BRCA1-deficient mammary tumor cells are dependent on EZH2 expression and sensitive to polycomb repressive complex 2-inhibitor 3-deazaneplanocin A. Breast Cancer Res. 2009;11(4):R63.
22. Shi B, Liang J, Yang X, et al. Integration of estrogen and Wnt signaling circuits by the polycomb group protein EZH2 in breast cancer cells. Mol Cell Biol. 2007;27(14):5105-5119.

23. Lee ST, Li Z, Wu Z, et al. Context-specific regulation of NF- $\kappa$ B target gene expression by EZH2 in breast cancers. Mol Cell. 2011;43(5):798-810.

24. Rao ZY, Cai MY, Yang GF, et al. EZH2 supports ovarian carcinoma cell invasion and/or metastasis via regulation of TGF- $\beta 1$ and is a predictor of outcome in ovarian carcinoma patients. Carcinogenesis. 2010;31(9):1576-1583.

25. Rizzo S, Hersey JM, Mellor P, et al. Ovarian cancer stem cell-like side populations are enriched following chemotherapy and overexpress EZH2. Mol Cancer Ther. 2011;10(2):325-335.

26. Li H, Bitler BG, Vathipadiekal V, et al. ALDH1A1 is a novel EZH2 target gene in epithelial ovarian cancer identified by genome-wide approaches. Cancer Prev Res (Phila). 2012;5(3):484-491.

27. Lu C, Han HD, Mangala LS, et al. Regulation of tumor angiogenesis by EZH2. Cancer Cell. 2010;18(2):185-197.

28. Kikuchi J, Kinoshita I, Shimizu Y, et al. Distinctive expression of the polycomb group proteins Bmil polycomb ring finger oncogene and enhancer of zeste homolog 2 in nonsmall cell lung cancers and their clinical and clinicopathologic significance. Cancer. 2010;116(12):3015-3024.

29. Huqun, Ishikawa R, Zhang J, et al. Enhancer of zeste homolog 2 is a novel prognostic biomarker in nonsmall cell lung cancer. Cancer. 2012;118(6):1599-1606.

30. Zhang $\mathrm{H}$, Zhang $\mathrm{H}$, Zhao $\mathrm{M}$, et al. MiR-138 inhibits tumor growth through repression of EZH2 in non-small cell lung cancer. Cell Physiol Biochem. 2013;31(1):56-65.

31. Piunti A, Pasini D. Epigenetic factors in cancer development: polycomb group proteins. Future Oncol. 2011;7(1):57-75.

32. Fiskus W, Wang Y, Sreekumar A, et al. Combined epigenetic therapy with the histone methyltransferase EZH2 inhibitor 3-deazaneplanocin $\mathrm{A}$ and the histone deacetylase inhibitor panobinostat against human AML cells. Blood. 2009;114(13):2733-2743.

33. Avan A, Crea F, Paolicchi E, et al. Molecular mechanisms involved in the synergistic interaction of the EZH2 inhibitor 3-deazaneplanocin A with gemcitabine in pancreatic cancer cells. Mol Cancer Ther. 2012;11(8):1735-1746.

34. McCabe MT, Ott HM, Ganji G, et al. EZH2 inhibition as a therapeutic strategy for lymphoma with EZH2-activating mutations. Nature. 2012;492(7427):108-112.
OncoTargets and Therapy

\section{Publish your work in this journal}

OncoTargets and Therapy is an international, peer-reviewed, open access journal focusing on the pathological basis of all cancers, potential targets for therapy and treatment protocols employed to improve the management of cancer patients. The journal also focuses on the impact of management programs and new therapeutic agents and protocols on

\section{Dovepress}

patient perspectives such as quality of life, adherence and satisfaction The manuscript management system is completely online and includes a very quick and fair peer-review system, which is all easy to use. Visit http://www.dovepress.com/testimonials.php to read real quotes from published authors. 